\title{
Glycine to tryptophan substitution in type I collagen in a patient with OI type III: a unique collagen mutation
}

\author{
Lieve Nuytinck, Turgut Tükel, Hülya Kayserili, Memnune Yüksel Apak, Anne De Paepe
}

Centre for Medical

Genetics, OK5,

University Hospital

Gent, 185 De

Pintelaan, B-9000

Gent, Belgium

L Nuytinck

A De Paepe

Institute of Child

Health, Medical

Genetics, University of

Istanbul, Istanbul,

Turkey

T Tükel

H Kayserili

M Y Apak

Correspondence to: Dr Nuytinck,

Lieve.Nuytinck@rug.ac.be

Revised version received

12 November 1999

Accepted for publication

22 December 1999

Table 1 The clinical features and mode of inheritance for all types of osteogenesis imperfecta are presented according to the classification of Sillence et al ${ }^{1}$

\begin{tabular}{|c|c|c|}
\hline OI type & Clinical features & Inheritance \\
\hline OI type I (mild) & $\begin{array}{l}\text { Normal or near normal stature } \\
\text { with little or no deformity } \\
\text { Variable number of fractures } \\
\text { Distinctly blue sclerae throughout } \\
\text { life } \\
\text { Hearing loss in about } 50 \% \text { of cases }\end{array}$ & $\mathrm{AD}$ \\
\hline OI type II (lethal) & $\begin{array}{l}\text { Lethal in perinatal period or in } \\
\text { utero } \\
\text { Severe dwarfism with marked } \\
\text { bowing and deformities of the } \\
\text { extremities } \\
\text { Extreme bone fragility }\end{array}$ & $\begin{array}{l}\mathrm{AD} \text { (new mutations) } \\
\mathrm{AR} \text { (rare) }\end{array}$ \\
\hline OI type III (severely deforming) & $\begin{array}{l}\text { Progressively deforming bones } \\
\text { Very short stature } \\
\text { Dentinogenesis imperfecta } \\
\text { common }\end{array}$ & $\begin{array}{l}\mathrm{AD} \\
\mathrm{AR} \text { (uncommon) }\end{array}$ \\
\hline OI type IV (moderately severe) & $\begin{array}{l}\text { Variable short stature } \\
\text { Mild to moderate bone deformity } \\
\text { Variable fracture rate } \\
\text { Scoliosis/lordosis in some patients } \\
\text { Dentinogenesis imperfecta } \\
\text { common }\end{array}$ & $\mathrm{AD}$ \\
\hline
\end{tabular}

AD: autosomal dominant; AR: autosomal recessive. the $\alpha 2$ (I) collagen chain. Glycine substitutions in type I collagen are the most frequent cause of the severe and lethal forms of OI. The phenotypic severity varies according to the nature and localisation of the mutation. Substitutions of glycine by tryptophan, which is the most voluminous amino acid, have not yet been identified in type I collagen or any other fibrillar collagen. The severe, though nonlethal OI phenotype associated with this mutation may appear surprising in view of the huge size of the tryptophan residue. The fact that the mutation resides within a so called "non-lethal" region of the $\alpha 2(\mathrm{I})$ collagen chain supports a regional model in phenotypic severity for $\alpha 2$ (I) collagen mutations, in which the phenotype is determined primarily by the nature of the collagen domain rather than the type of glycine substitution involved.

$(\mathfrak{M}$ Med Genet 2000;37:371-375)
Keywords: osteogenesis imperfecta; COL1A2; tryptophan; collagen

We report a unique glycine substitution in I collagen and highlight the clinical and biochemical consequences. The proband is a 9 year old Turkish boy with severely deforming osteogenesis imperfecta (OI). Biochemical analysis of (pro) collagen type I from a skin fibroblast culture showed both normal and overmodified $\alpha$ chains. Molecular analysis showed a $G>T$ transversion in the COL1A2 gene, resulting in the substitution of glycine by tryptophan at position 277 of

Osteogenesis imperfecta (OI) is a heritable connective tissue disorder characterised by a varying degree of bone fragility. Generally, four major clinical and genetic subtypes are recognised (table 1). ${ }^{1}$

OI is caused by mutations in one of the two $\alpha$ chains of type I collagen, the principal component of the extracellular matrix in bone and skin. Type I collagen is a fibrillar collagen composed of two $\alpha 1$ (I) chains, encoded by the COL1A1 gene, and one $\alpha 2(\mathrm{I})$ chain, encoded by the COL1A2 gene. In the majority of patients with mild OI a decreased amount of structurally normal type I collagen is found whereas in the more severe OI phenotypes (OI types II, III, and IV) a structurally abnormal type I collagen protein is seen. Most commonly, a structural defect is caused by the substitution of a glycine residue in the helical part of one or both type I collagen $\alpha$ chains. In the vast majority of cases, the mutations are "private" in that they have only been reported in one single person/family.

Glycine substitutions are by far the most frequently found amino acid alteration not only in type I collagen but also in the other collagen types known to form fibrils. Fibrillar collagens have an uninterrupted triple helical domain constructed from repeating Gly-X-Y triplets. The presence of glycine, the amino acid with the smallest side chain, in every third position of a collagen $\alpha$ chain is a prerequisite for correct folding of the three $\alpha$ chains into a collagen triple helix.

Here we present the clinical and biochemical consequences of a glycine to tryptophan substitution in the procollagen $\alpha 2(\mathrm{I})$ chain in a patient with OI type III. Tryptophan, an amino acid residue which is not usually present in the type I collagen triple helical region, consists of an aromatic side chain composed of an indol ring bound by a methylene group. The result is a huge, neutral amino acid with a non-polar side chain, as such being very hydrophobic. The mutation presented here is unique in that it is the first reported tryptophan for glycine substitution in type I collagen causing OI.

\section{Materials and methods}

CLINICAL SUMMARY

The proband is a 9 year old Turkish boy, the only child of a non-consanguineous couple. The father and mother were 33 and 28 years old respectively at the time of his birth. They 

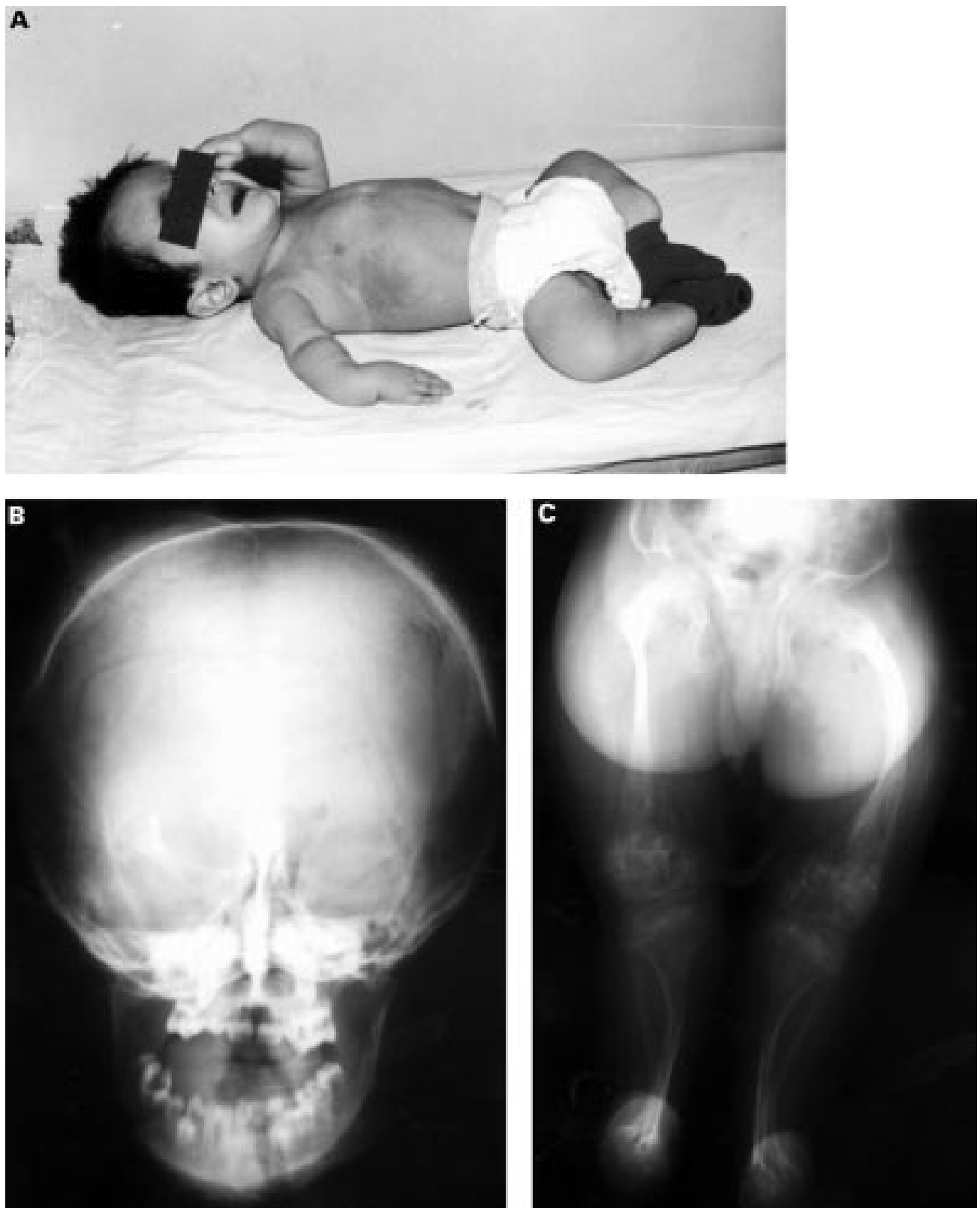

Figure 1 (A) The proband at the age of 6 years, showing severe dwarfism and deformities of the upper and lower extremities, indicative of the severely deforming type of OI (Sillence type III). (B) X ray of the skull at the age of 9 years showing thin, undermineralised calvarium and wormian bones. $(C) X$ rays of the lower extremities taken at the age of 9 years, showing severe osteoporosis, thinning and bowing of the long bones, and the characteristic "popcorn" deformities in the metaphyseal regions of the femora and tibiae.

are healthy and have no history of fractures. At birth, the proband weighed $2300 \mathrm{~g}$ and had several fractures. His length was not recorded. Thereafter, he suffered countless other fractures, mainly of the extremities and also of the ribs. He was never able to walk or sit alone. At the age of 6 years 3 months, he was severely dwarfed with a length of $72 \mathrm{~cm}$ (below the $3 \mathrm{rd}$ centile) (fig 1A) and a head circumference of $49.5 \mathrm{~cm}$. He had blue sclerae, pectus carinatum, severely deformed limbs, and dentinogenesis imperfecta. Now, at the age of 9 years, his fracture rate is still high, his limb deformities have progressed, and his general condition has worsened. His height is at present $83 \mathrm{~cm}$ (below the 3 rd centile). $X$ rays show, besides generalised osteoporosis, a large skull with irregular and undermineralised calvarium and wormian bones (fig 1B), thin ribs with old fractures, a severely deformed pelvis, and thin and markedly deformed long bones in the upper and lower extremities with popcorn deformities in the metaphyseal regions of the long bones (fig 1C). The clinical and radiological features correspond to the severely progressive type of OI (Sillence type III).

BIOCHEMICAL COLLAGEN STUDIES

A skin biopsy was obtained from the proband and a fibroblast culture was established under standard conditions. After labelling with ${ }^{14} \mathrm{C}$ proline, the procollagen and collagen proteins were isolated from the medium and cell layer and examined by SDS electrophoresis as described by Nuytinck et al. ${ }^{2}$ Thermal stability measurement was performed as described by 
Bruckner and Prockop. ${ }^{3}$ The collagen processing and secretion were studied by pulsed chase experiments adapted from Forlino et $a .^{4}{ }^{4}$ Confluent cells were labelled with ${ }^{14} \mathrm{C}$-proline for 30 minutes. Radiolabelled medium was removed and replaced with serum-free BME supplemented with ascorbic acid and 10 $\mathrm{mmol} / 1$ cold proline. Cells were chased for 1 hour, 1.5 hours, 2 hours, 3.5 hours, 5 hours, and 10.5 hours. At the indicated times, the medium and cell layer were harvested separately and supplemented with protein inhibitors. The samples were run reduced without pepsin digestion on SDS-PAGE.

MOLECULAR COLLAGEN STUDIES

Genomic DNA of the parents was isolated from peripheral blood leucocytes using the Qiagen-Blood miniprep kit (Qiagen Inc, Chatworth, CA). Genomic DNA from the proband was isolated from dermal fibroblasts using the Easy-DNA kit (Invitrogen). Total RNA was isolated from cultured fibroblasts and cDNA was obtained, using M-MLV reverse transcriptase (Life Technologies) according to the manufacturer's instructions. PCR was performed using primers for the complete $C O L 1 A 1$ and $C O L 1 A 2$ coding regions and the amplimers were analysed by conformation sensitive gel electrophoresis (CSGE). ${ }^{5}$ Fragments showing an aberrant migration pattern were cloned and sequenced. The RT-PCR findings were confirmed by genomic DNA sequencing.

\section{Results}

BIOCHEMICAL COLLAGEN STUDIES

Polyacrylamide gel electrophoresis (PAGESDS) of procollagen and pepsin digested collagens from the medium and from the cell layer showed a population of normal and of more slowly migrating (pro) $\alpha 1$ (I) and (pro) $\alpha 2(\mathrm{I})$ collagen chains (fig 2). The collagen type I melting profiles showed a slight decrease of $1^{\circ} \mathrm{C}$ to $2^{\circ} \mathrm{C}$ in denaturing temperature between the mutant and normal type I collagen chains (data not shown). Pulse chase experiments showed only a mild delay in processing and secretion in comparison to a normal control cell line (data not shown).

MOLECULAR COLLAGEN STUDIES

Molecular screening by CSGE showed one migration shift in the COL1A2 gene fragment covering exon 19 to exon 24 in the proband's cDNA. Cloning and sequencing of the relevant COL1A2 fragment on cDNA as well as on genomic DNA resulted in the identification of a $\mathrm{G}$ to $\mathrm{T}$ transversion in position 1238 of the COL1A2 cDNA sequence (EMBL accession number Z74616), which changes the codon for glycine (GGG) to that for tryptophan (TGG) (fig 3) in position 277 of the proa2(I) collagen chain. Restriction digestion with the enzyme ApaI of the respective amplimers confirmed the presence of the mutation in cDNA and genomic DNA in the proband and the absence of the mutation in genomic DNA from both parents.

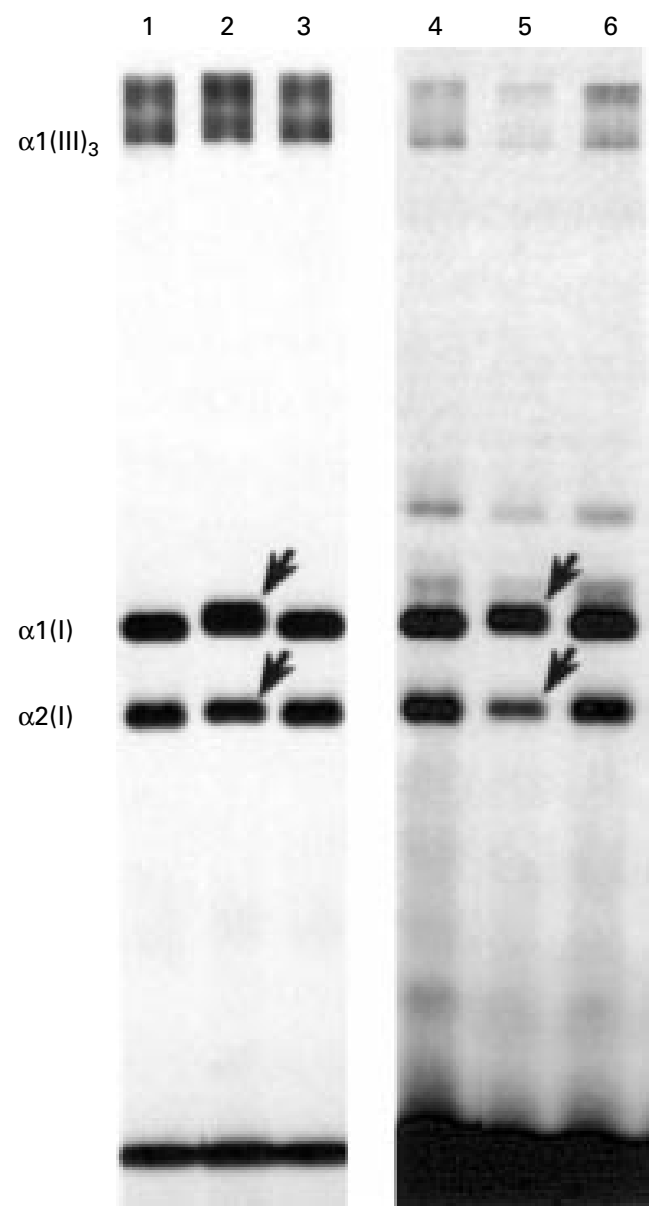

Figure 2 Analysis by SDS-PAGE of pepsin derived collagens secreted in the medium (left panel) and collagens retained in the cell layer (right panel) by cultured fibroblasts from the proband compared to control samples. Lanes 1, 3, 4, and 6: control samples; lanes 2 and 5: the proband with OI type III. Broadening of the bands (arrows) representing the $\alpha 1$ (I) and a2(I) collagen chains is visible in the medium as well as in the cell layer. This is because of the presence of two different populations of type I collagen a chains, a normal and a mutant, more slowly migrating population. In the medium as well as in the cell layer, both populations are seen in equal proportions.

\section{Discussion}

The substitution of a glycine residue by a bulkier amino acid in type I collagen is a common mechanism in OI. Of the 20 naturally occurring amino acids, mutations in a codon for glycine (GGT, GGC, GGA, GGG) can result in the substitution by only a limited number of amino acids. Base changes in the first position can give rise to arginine, cysteine, serine, tryptophan, or a stop codon. Any base change occurring in the second position results in the substitution of the glycine residue by alanine, aspartic acid, glutamic acid, or valine. All of these amino acid substitutions have been found in both type I collagen genes, except two, namely glycine to tryptophan and glycine to a stop codon.

The preferential occurrence of a certain amino acid residue as a result of a base change in the codon for glycine is in part determined by the relative codon usage of the glycine codon (table 2). The tryptophan codon (TGG) can only arise from the codon GGG, which is the codon with the lowest prevalence in either 


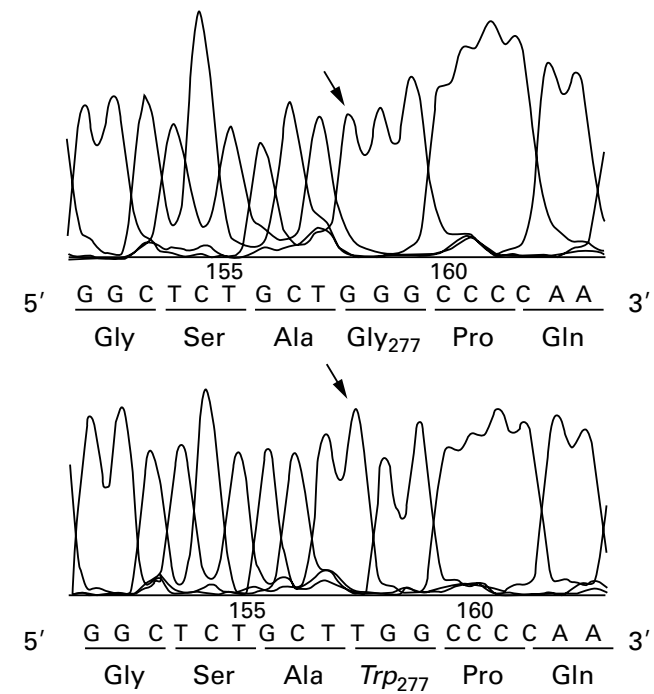

Figure 3 Sequencing data of the normal and the mutant COL1A2 allele in the OI type III patient. A $G$ to $T$ transversion changing the codon for Gly (GGG) in position 277 of the proa2 (I) collagen chain to the codon for Trp (TGG) is seen (arrow).

Table 2 The codon usage for the amino acid glycine (\%) in both type I collagen genes (COL1A1 and COL1A2) is given, illustrating the scarcity of the GGG codon from which the tryptophan residue arises

\begin{tabular}{lllll}
\hline & $G G T$ & $G G C$ & $G G A$ & $G G G$ \\
\hline COL1A1 & 51 & 27 & 18 & 3 \\
COL1A2 & 52 & 22 & 21 & 5 \\
\hline
\end{tabular}

the COL1A1 or COL1A2 gene. This may be one reason why a glycine by tryptophan substitution has not been reported previously in OI. ${ }^{6}$ Similarly, glutamic acid residues (GAG), which also arise from the codon GGG, have only been reported once in the COL1A1 gene (Nuytinck and De Paepe, personal communication) and once in the COL1A2 gene. ${ }^{7}$ Until now, only the substitution of GGA by TGA resulting in a stop codon has not been found in type I collagen genes. Although nonsense mutations in the COL1A1 gene are common in OI type I, they arise in all cases from the substitution of arginine for a stop codon (AGA or CGA to TGA) in the $\alpha 1$ (I) collagen helix.

The $\alpha 2(\mathrm{I})-\mathrm{G} 277 \mathrm{~W}$ substitution reported here is localised in a microdomain of the type I collagen molecule in which some glycine substitutions have been characterised (fig 4). All of the $\alpha 1(\mathrm{I})$ collagen mutations described in this region result in lethal phenotypes while in the $\alpha 2(\mathrm{I})$ chain they result in non-lethal phenotypes. From these data, in addition to the $\alpha 2(\mathrm{I})-\mathrm{G} 277 \mathrm{~W}$ substitution reported here, one can conclude that the region in the $\alpha 2(\mathrm{I})$ collagen chain in which the mutation occurs rather than the type of the substituting amino acid is predominant in determining the clinical outcome. As this is the first report of a glycine to tryptophan substitution in type I collagen, one can speculate that a similar substitution either in the $\alpha 1(\mathrm{I})$ or in other regions along the $\alpha 2$ (I) chain would result in a lethal OI phenotype.

On biochemical analysis, one can easily observe the presence of normal and $\alpha 1(\mathrm{I})$ $\alpha 1(\mathrm{I})$

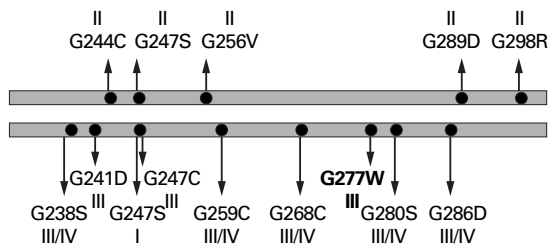

Figure 4 Overview of the published glycine substitutions identified in both type I collagen a chains in a region from amino acid residue 230 to 300 . The position and nature of the glycine substitutions and the resulting clinical phenotypes are given. a1(I) collagen chain: G244C, ${ }^{9}$ G247S, ${ }^{10}$ G256V, ${ }^{11}$ G289D (Nuytinck and De Paepe, personal communication), G298R. ${ }^{12}$ a2(I) collagen chain: G238S, ${ }^{8}$ G241D, ${ }^{13}$ G247S, ${ }^{14}$ G247C, ${ }^{15}$ G259C, ${ }^{16}$ G268C, ${ }^{17} \mathrm{G} 280 S,{ }^{17} \mathrm{G} 286 \mathrm{D} .{ }^{17}$

chains, a more slowly migrating population of $\alpha 1(\mathrm{I})$ chains, and also $\alpha 2(\mathrm{I})$ chains in the medium, which means that they are apparently well secreted. There is no significant accumulation of mutant $\alpha$ chains in the cell layer. Thermal stability measurements showed a mild decrease in denaturing temperature between the mutant and normal type I collagen trimers. These findings illustrate that a change in thermal stability does not necessarily influence the clinical outcome as already suggested earlier. ${ }^{8}$ Pulse chase experiments showed only a slight delay in processing and secretion of the type I collagen molecules, a phenomenon which is often observed in OI patients with a glycine substitution in type I collagen. As such, the in vitro effect of the glycine by tryptophan substitution on the molecular secretion and thermal stability is quite mild, taking into account that tryptophan is such a huge amino acid residue. One explanation for these findings is the $\mathrm{N}$-terminal localisation of the tryptophan residue in the $\alpha 2$ (I) collagen chain. As helix folding of type I collagen molecules proceeds from the C- to the N-terminal propeptide, only the winding of the helical region N-terminal to the substitution will be impaired.

Since each type I collagen molecule consists of two $\alpha 1$ (I) chains and one $\alpha 2$ (I) chain, which is the one mutated in this patient, half of the type I collagen molecules produced by this patient are expected to be normal while the other half will be structurally defective. Since no evidence was found in vitro for accumulation of mutant type I collagen molecules in the cell layer, one can assume that both normal and mutant molecules are present in equal proportions in the extracellular matrix and available for participation in collagen fibril formation.

We are grateful to $\mathrm{K}$ Wettinck, $\mathrm{M}$ Van Thielen, and $\mathrm{N}$ Aslan for excellent technical assistance. This work was supported by the FWO-Vlaanderen (Fund for Scientific Research, Flanders).

1 Sillence DO, Senn A, Danks DM. Genetic heterogeneity in osteogenesis imperfecta. F Med Genet 1979;16:101-16.

2 Nuytinck L, Narcisi P, Nicholls A, Renard JP, Pope FM, De Paepe A. Detection and characterisation of an overmodified type III collagen by analysis of non-cutaneous connective tissues in a patient with Ehlers-Danlos syndrome IV. 7 Med Genet 1992;29:375-80.

3 Bruckner P, Prockop DJ. Proteolytic enzymes as probes for the triple-helical conformation of procollagen. Anal Biochem $1981 ; 110: 360-8$

4 Forlino A, Zolezzi F, Valli M, et al. Severe (type III) osteogenesis imperfecta due to glycine substitutions in the central domain of the collagen triple helix. Hum Mol Genet 1994;3:2201-6. 
5 Ganguly A, Rock MJ, Prockop DJ. Conformation-sensitive gel electrophoresis for rapid detection of single-base differences in double-stranded PCR products and DNA fragments: evidence for solvent-induced bends in DNA heteroduplexes. Proc Natl Acad Sci USA 1993;90:10325-9.

6 Dalgleish R. The human collagen mutation database 1998 Nucleic Acids Res 1998;26:253-5.

7 Rose NJ, Mackay K, Byers PH, Dalgleish R. A novel glycine to glutamic acid substitution at position 343 in the $\alpha 2$ chain of type I collagen in an individual with lethal osteogenesis imperfecta. Hum Mol Genet 1993;2:2175-7.

8 Nuytinck L, Wettinck K, Freund M, Van Maldergem L, Fabry G, De Paepe A. Osteogenesis imperfecta phenotypes resulting from serine for glycine substitutions in the $\alpha 2$ (I) collagen chain. Eur f Hum Genet 1997;5:161-7.

9 Fertala A, Westerhausen A, Morris G, Rooney JE, Prockop DJ. Two cysteine substitutions in procollagen I: a glycine replacement near the N-terminus of $u$ (1) chain causes the $\alpha 2$ (I) chain markedly destabilizes the triple helix. the $\alpha 2$ (I) chain markedly

10 Mackay K, Byers PH, Dalgleish R. An RT-PCR-SSCP screening strategy for detection of mutations in the gene encoding the $\alpha 1$ chain of type I collagen: application to four patients with osteogenesis imperfecta. Hum Mol Genet 1993;2:1155-60.

11 Patterson E, Smiley E, Bonadio J. RNA sequence analysis of a perinatal lethal osteogenesis imperfecta mutation. F Biol Chem 1989;264:10083-7.
12 Byers PH, Wallis GA, Willing MC. Osteogenesis imperfecta: translation of mutation to phenotype. $f$ Med Genet 1991;28:433-42.

13 Lund AM, Astrom E, Soderhall S, Schwartz M, Skovby F. Osteogenesis imperfecta: mosaicism and refinement of the genotype-phenotype map in OI type III. Mutations in brief No 242. Hum Mutat 1999;13:503.

14 Zhuang J, Tromp G, Kuivaniemi H, Castells S, Bugge M, Prockop DJ. Direct sequencing of PCR products derived from cDNAs for the pro $\alpha 1$ and pro $\alpha 2$ chains of type I procollagen as a screening method to detect mutations in patients with osteogenesis imperfecta. Hum Mutat 1996;7: 89-99.

15 Marini JC, Wang Q, Filie JD, Lewis MB. Mutations in $\alpha 2(\mathrm{I})$ collagen support a regional model of the relationship between osteogenesis imperfecta genotype and phenotype. Fifth International Conference on OI, 1993:126.

16 Wenstrup RJ, Shrago-Howe AW, Lever LW, Phillips CL, Byers PH, Cohn DH. The effects of different cysteine for glycine substitutions within alpha2(I) chains. Evidence of distinct structural domains within the type I collagen triple helix. F Biol Chem 1991;266:2590-4.

17 Lund AM, Nicholls AC, Schwartz M, Skovby F. Parental mosaicism and autosomal dominant mutations causing structural abnormalities of collagen I are frequent in families with osteogenesis imperfecta type III/IV. Acta Paediatr 1997;86:711-18. 